\title{
The Global-First Topological Definition of Perceptual Objects, and Its Neural Correlation in Anterior Temporal Lobe
}

\author{
Lin Chen, Ke Zhou, Wenli Qian, and Qianli Meng \\ State Key Laboratory of Brain and Cognitive Science \\ Institute of Biophysics, Chinese Academy of Sciences \\ 15 Datun Road, 100101 Beijing, China \\ linchen@bcslab.ibp.ac.cn
}

What is a perceptual object? This question seems to be straightforward yet its answer has become one of the most central and also controversial issues in many areas of cognitive sciences.

The "global-first" topological approach ties a formal definition of perceptual objects to invariance over topological transformation, and the core intuitive notion of a perceptual object - the holistic identity preserved over shape-changing transformations - may be precisely characterized as topological invariants, such as connectivity and holes.

The topological definition of objects has been verified by a fairly large set of behavioral experiments, including, for example, MOT and attention blink, which consistently demonstrated that while object identity can survive various non-topological changes, the topological change disturbs its object continuity, being perceived as an emergence of a new object. Companion fMRI experiments revealed the involvement of anterior temporal lobe, a late destination of the visual form pathway, in the topological perception and the formation of perceptual objects defined by topology. This contrast of global-first in behavior and late destination in neuroanatomy raises far-reaching issues regarding the formation of object representations in particular, and the fundamental question of "where to begin" in general. 